\title{
Structure of dense adsorption layers of escin at the air-water interface studied by molecular dynamics simulations
}

\author{
Sonya Tsibranska, ${ }^{\dagger}$ Anela Ivanova, ${ }^{*},{ }^{\star}$ Slavka Tcholakova, ${ }^{\dagger}$ Nikolai Denkov ${ }^{\dagger}$ \\ ${ }^{\dagger}$ Department of Chemical and Pharmaceutical Engineering, and ${ }^{*}$ Department of Physical Chemistry, \\ Faculty of Chemistry and Pharmacy, University of Sofia, I James Bourchier Ave., 1164 Sofia, Bulgaria
}

(Supporting Information) 


\section{Analysis of the total number of hydrogen bonds}

Figure S1A presents the evolution of the number of hydrogen bonds within and between escin molecules over time for the two studied models. It is clearly seen that there is a trend for decrease of the number of $\mathrm{H}$-bonds with time in both layers. This is probably due to the fact that the number of H-bonds is influenced substantially by the intermolecular orientation. It means that the molecules try to orient with respect to each other in time in a way, which minimizes the steric hindrance and, hence, the energy. It is also evident from the plot that after $150 \mathrm{~ns}$ the number of $\mathrm{H}$-bonds reaches a constant average value in both models. This is then the characteristic relaxation time for models of this size (49 molecules). To determine the rate of increase of the number of $\mathrm{H}$-bonds, the data from Figure S1A are fit to straight lines separately before and after $150 \mathrm{~ns}$. The linear regression coefficient is not high because of the presence of short-lived but intensive fluctuations in the data. However, the general trends in the two parts of the trajectories are close to linear. Therefore, the obtained slope of the initial straight line shows that the number of hydrogen bonds decreases by 0.2 per ns in both models. The very similar slopes in the two systems illustrate the drive to form optimal number of hydrogen bonds, independent of the surface concentration.

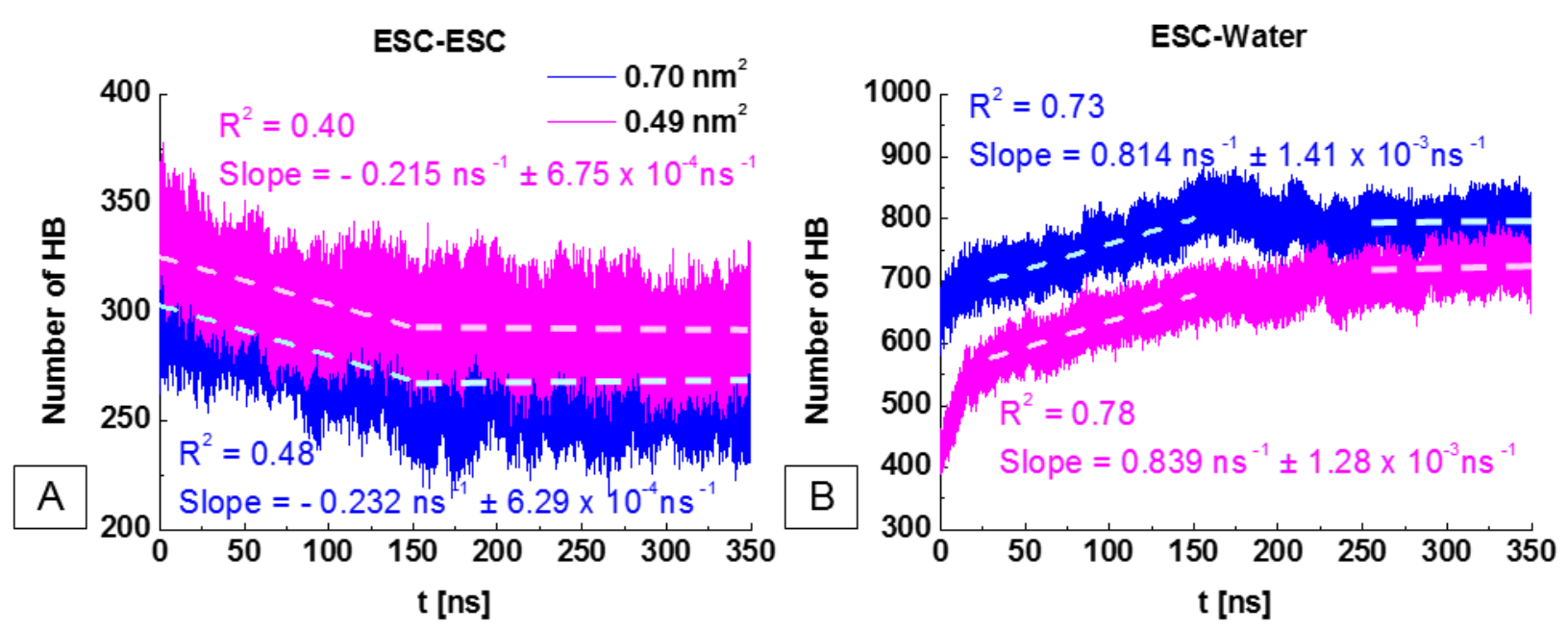

Figure S1. Number of hydrogen bonds (A) in and between escin molecules and (B) between escin and water molecules as a function of time.

It turns out that the number of hydrogen bonds escin-water (Figure S1B) increases with time for both models concurrently to the decrease of inter-surfactant H-bonds. This decrease can be attributed to the attempt of water molecules to penetrate and hydrate the hydrophilic heads of escin molecules. The process continues until equilibrium degree of hydration is reached. The linear fit of the initial part of the trajectories yields about four times larger slope compared to that of ESC-ESC bonds for both models. This outlines that the process of hydration progresses much faster than that of surfactant reorientation. 
A plateau is reached also in the ESC-water profiles after $150 \mathrm{~ns}$, confirming the relaxation time for achieving optimum H-bonding in the studied models.

\section{Density profiles}

Table S1. $\mathrm{R}^{2}$-coefficient, standard deviation $(\sigma)$, and FWHM for the Gaussian fits of the escin mass density profiles.

\begin{tabular}{|c|c|c|}
\hline Model & 49ESC-70 & 49ESC-49 \\
\hline $\mathbf{R}^{\mathbf{2}}$ & 0.985 & 0.995 \\
\hline $\boldsymbol{\sigma}, \mathbf{n m}$ & 0.93 & 1.27 \\
\hline FWHM, nm & 2.19 & 3.00 \\
\hline
\end{tabular}

Table S2. Escin layer thickness normal to the interface estimated after fixing the minimum density at one, two, or three standard deviations away from the Gaussian maximum; the two numbers - for escin and water density - in each cell correspond to the values on the left and right side of the curve in Figure 3 of the manuscript, respectively.

\begin{tabular}{|c|c|c|c|c|c|c|}
\hline \multirow[t]{3}{*}{ Model } & \multicolumn{3}{|c|}{ 49ESC-70 } & \multicolumn{3}{|c|}{ 49ESC-49 } \\
\hline & Layer & $\begin{array}{c}\text { Escin } \\
\text { density }\end{array}$ & $\begin{array}{l}\text { Water } \\
\text { density }\end{array}$ & Layer & $\begin{array}{c}\text { Escin } \\
\text { density }\end{array}$ & $\begin{array}{l}\text { Water } \\
\text { density, }\end{array}$ \\
\hline & $\begin{array}{c}\text { thickness, } \\
\text { nm }\end{array}$ & $\mathrm{kg} / \mathrm{m}^{3}$ & $\mathrm{~kg} / \mathrm{m}^{3}$ & $\begin{array}{c}\text { thickness, } \\
\text { nm }\end{array}$ & $\mathrm{kg} / \mathrm{m}^{3}$ & $\mathrm{~kg} / \mathrm{m}^{3}$ \\
\hline \multirow{2}{*}{$1 \sigma$} & \multirow{2}{*}{1.86} & 705 & 528 & \multirow{2}{*}{2.55} & 716 & 489 \\
\hline & & 849 & 121 & & 807 & 180 \\
\hline \multirow{2}{*}{$2 \sigma$} & \multirow{2}{*}{3.73} & 106 & 920 & \multirow{2}{*}{5.09} & 89 & 923 \\
\hline & & 102 & 11 & & 148 & 25 \\
\hline \multirow{2}{*}{$3 \sigma$} & \multirow[b]{2}{*}{5.59} & 13 & 972 & \multirow{2}{*}{7.64} & 3 & 980 \\
\hline & & 29 & 4 & & 9 & 2 \\
\hline
\end{tabular}




\section{Interfacial tension isotherms:}

The empirical models used to extract the proper surface tension for comparison with the computed value are as follows:

$$
\begin{gathered}
\frac{\pi}{K_{B} \mathrm{~T} \Gamma_{\infty}}=\frac{\Theta}{1-\Theta} \\
K_{a d s} C=\frac{\Theta}{1-\Theta} \exp \left(\frac{\Theta}{1-\Theta}\right) \\
K_{a d s} C=\frac{\pi}{K_{B} \mathrm{~T} \Gamma_{\infty}} \exp \left(\frac{\pi}{K_{B} \mathrm{~T} \Gamma_{\infty}}\right) \\
\frac{\pi}{K_{B} \mathrm{~T} \Gamma_{\infty}}=\frac{\Theta}{1-\Theta}-\tilde{\beta} \Theta^{2} \\
K_{a d s} C=\frac{\Theta}{1-\Theta} \exp \left(\frac{\Theta}{1-\Theta}-2 \tilde{\beta} \Theta\right) \\
\tilde{\beta}=\frac{\beta \Gamma_{\infty}}{K_{B} \mathrm{~T}}
\end{gathered}
$$

There, $\mathrm{k}_{\mathrm{B}}$ is the Boltzmann constant, $\mathrm{T}$ is the absolute temperature, $\mathrm{C}$ is the surfactant concentration in the bulk solution, $\Theta$ is the surfactant adsorption (surface concentration), $\Gamma_{\infty}$ is the maximum adsorption in the respective dense adsorption monolayer, $\mathrm{K}_{\mathrm{ads}}$ is the adsorption constant, and $\beta$ is the parameter in the van der Waals isotherm, which accounts for the interactions between two adsorbed molecules.

For correct estimation of the bulk concentration corresponding to the surface concentration in the models we used some of the parameters obtained when processing the surface tension isotherm measured experimentally by Pagureva et al. [Ref. 12 of the main manuscript]: maximum adsorption $\left(\Gamma_{\infty}\right)$, adsorption constant $\left(\mathrm{K}_{\mathrm{ads}}\right)$ and interaction parameter for the van der Waals isotherm $(\beta)$. This bulk concentration is $0.001 \mathrm{wt} . \%$ with parameters from the Volmer isotherm and 0.0004 wt. \% using those of the van der Waals model. 


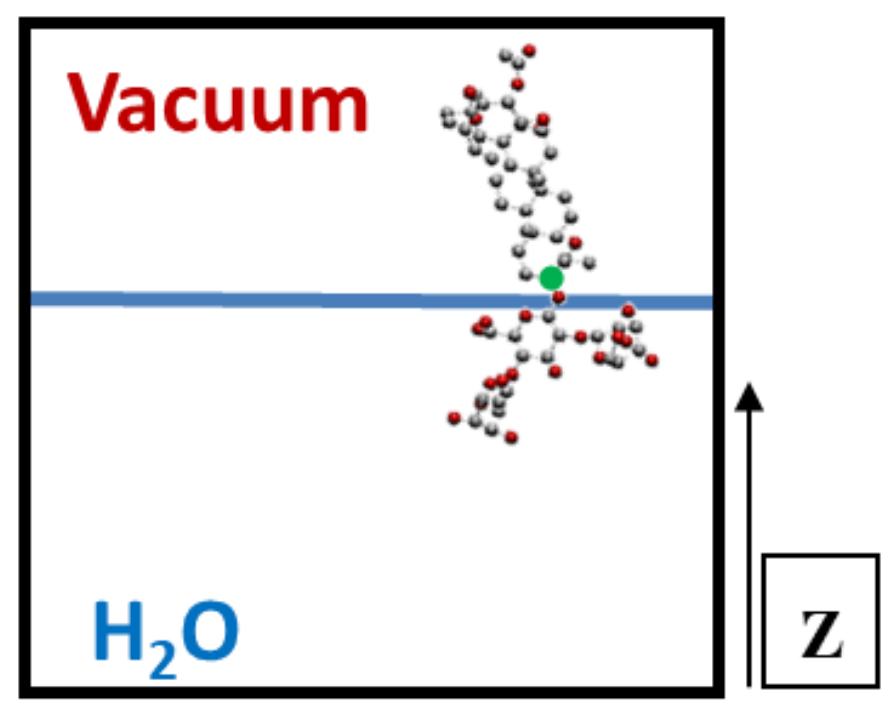

Figure S2. Schematic representation of the selected reference carbon atom (marked in green) from the aglycone of escin.

A
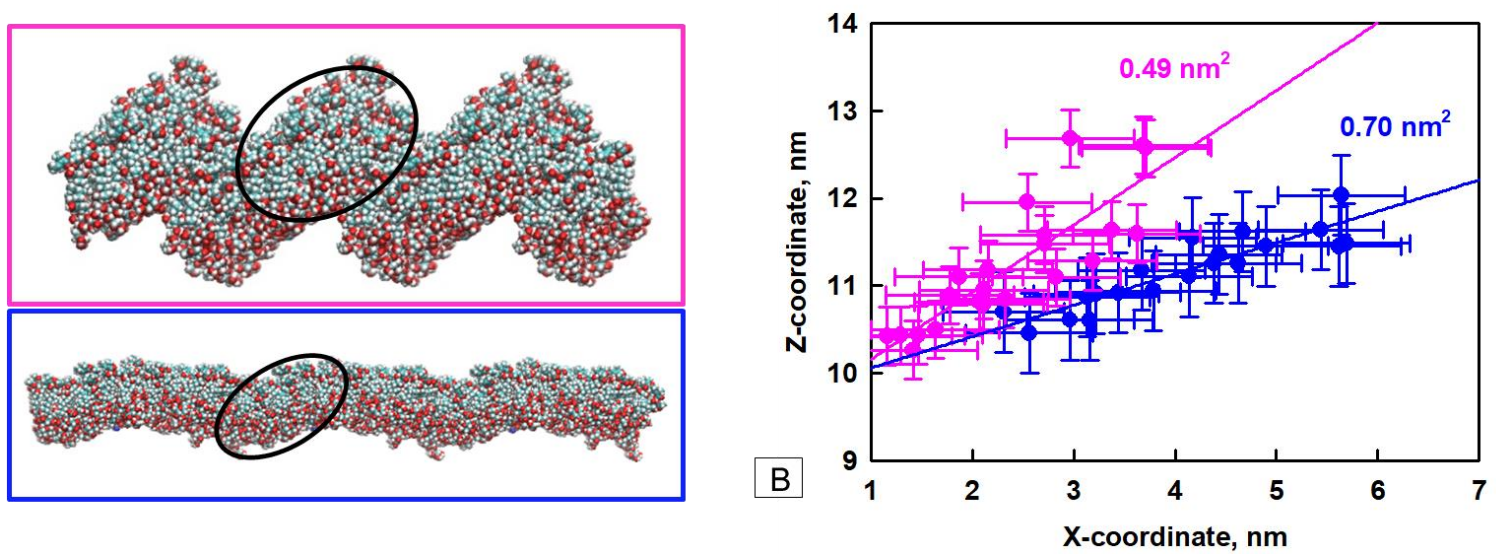

Figure S3. Average $\mathrm{z}$-coordinates as a function of the average $\mathrm{x}$-coordinates of the atom $\mathrm{C} 19$ of all ESC molecules in the models 49ESC-70 (blue) and 49ESC-49 (magenta); the solid lines denote the linear fits to the quasilinear parts of the profiles shown in A.

The average z-coordinate as a function of the average x-coordinate of each ESC molecule during the last $50 \mathrm{~ns}$ of each trajectory is traced. We fit the quasilinear part of the data for both models to a straight line shown in Figure S3. From the fit we determine an average slope of the surface relative to the z-axis. From this slope we calculate the correction for the surfactant tilt angle (see main manuscript). 

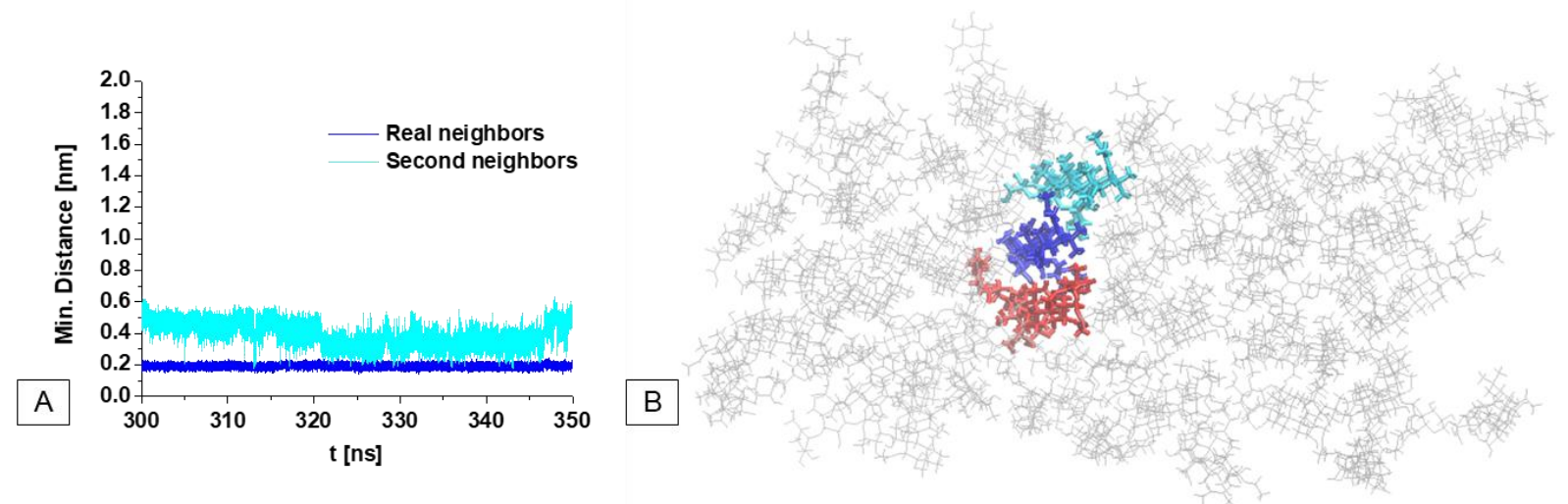

Figure S4. (A) Evolution of the minimum distance and (B) visual representation of the position of illustrative escin molecules, which are actual neighbors (blue) or second neighbors (cyan) to the red molecule.

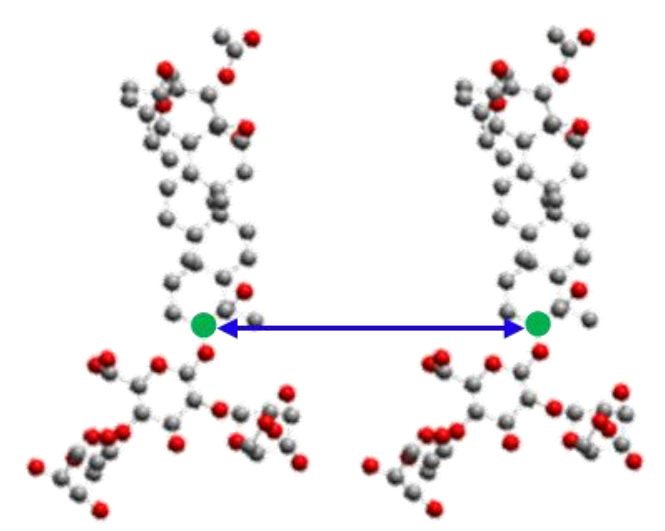

Figure S5. Schematic representation of the distance between the reference carbon atoms of escin used for calculation of the RDFs.
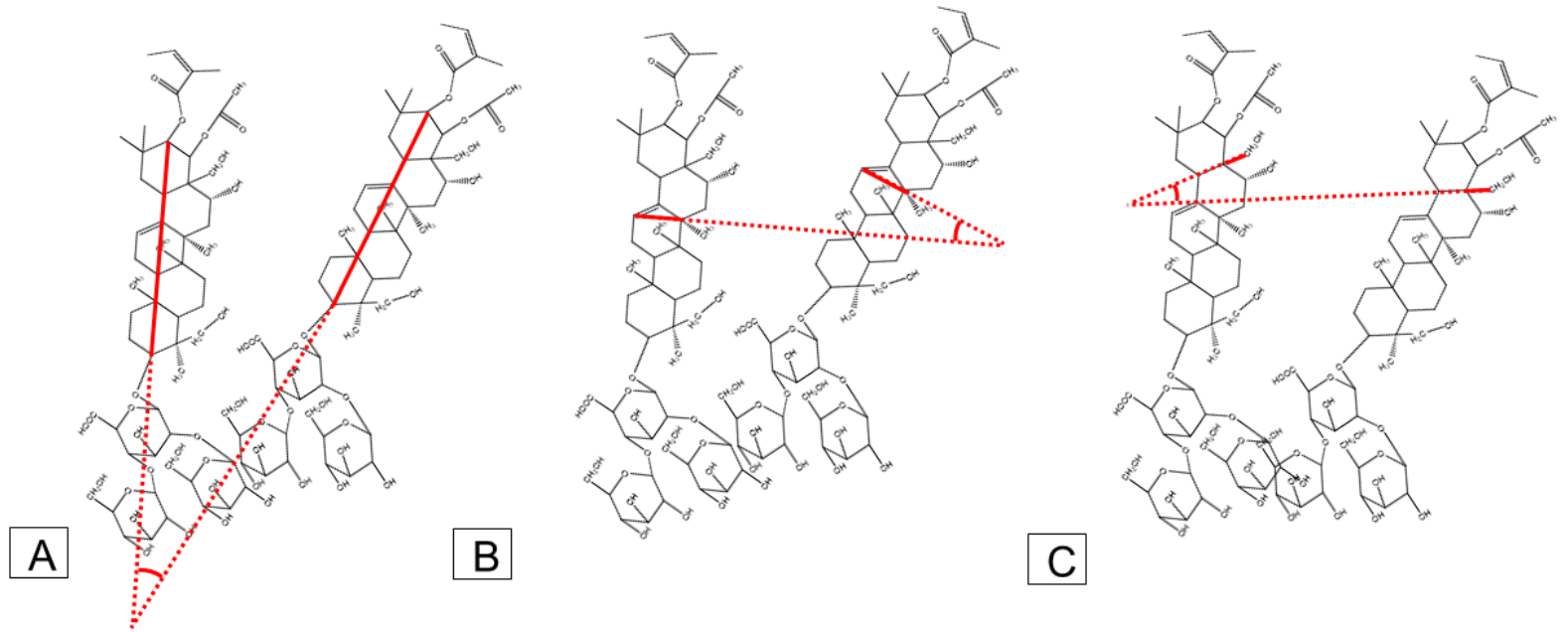

Figure S6. Schematic representation of the monitored angles closed between three quasiperpendicular vectors in the aglycones (A: $\alpha$; B: $\alpha^{\prime}$; C: $\alpha^{\prime \prime}$ ) for the model 49 ESC-70. 
Figure S6 contains the schematic representation of three complementary angles, closed between identical vectors in neighboring ESC molecules, which provide complete quantification of the mutual orientation of these surfactants. The three vectors in each molecule are perpendicular to each other (with deviations smaller than $5^{\circ}$ ).

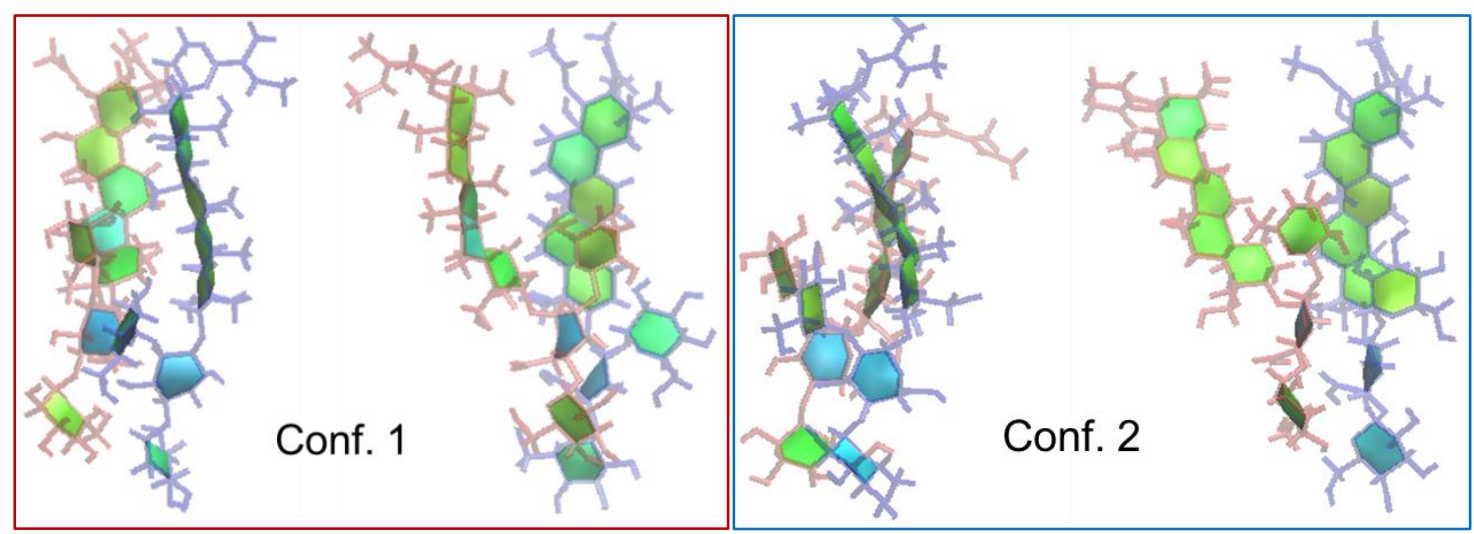

Figure S7. Visual representation of the position of two escin molecules from the model 49 ESC-70, which are actual neighbors and undergo a screw-type displacement (Conf. 1 to Conf. 2 and back); side view (left in each panel) and front view (right in each panel).

The evolution of their values along the MD trajectories is indicative of the presence of a screw-type motion. The two different intermolecular alignments, resulting from it, are illustrated in Figure S7 and the values of the three angles for them are summarized in Table S3. It may be seen that in configuration 1 the aglycones are almost perpendicular to each other in the direction of angle , $\alpha^{\prime \prime \prime}$, while in configuration 2 they are more parallel in this direction. The angles , $\alpha$ " and " $\alpha$ "” also change upon the transition, even though less, confirming that this is a screw-type displacement.

Table S3. Values of the three angles for the two configurations shown in Figure S7.

\begin{tabular}{|c|c|c|c|}
\hline Angle, $^{\circ}$ & $\alpha$ & $\alpha^{\prime}$ & $\alpha^{\prime \prime}$ \\
\hline Configuration 1 & 25 & 72 & 69 \\
\hline Configuration 2 & 39 & 44 & 52 \\
\hline
\end{tabular}

